\title{
L'histoire de la santé dans le pays de Vaud au Moyen Age: un bilan
}

par Jean-Daniel Morerod et Agostino Paravicini Bagliani

\section{RÉsumé}

Les sources laissées par les institutions médiévales vaudoises sont assez riches pour aborder dans ses aspects les plus divers l'histoire de la santé. Des études récentes traitent de la gestion du patrimoine viticole de l'hôpital de Vevey, des personnes qui y furent hospitalisées au début du $X V^{e}$ siècle, de la réaction des hôpitaux aux afflux massifs de pèlerins ou de victimes de catastrophes. La progression d'une épidémie - les pestes de 1348 et de 1360 - et son impact démographique ont pu être précisés, tandis qu'on dessinait un réseau des léproseries vaudoises. Enfin, l'attitude des bourgeois de Lausanne envers l'hôpital se dégage des legs pieux-étudiés pour le XIVe siècle-et, sous un tout autre angle, des prétentions politiques au contrôle de ces établissements.

L'histoire de la santé dans le Pays de Vaud médiéval jouit d'un statut privilégié. Des recherches menées pendant plusieurs décennies par un médecin historien, Eugène Olivier (1868-1955) ont conduit à la publication de Médecine et santé dans le Pays de Vaud des origines au XVIII ${ }^{e}$ siècle ${ }^{1}$, ouvrage somme toute exemplaire pour l'époque et extraordinairement ouvert à tous les aspects de l'histoire de la médecine et de la santé: prosopographie du milieu médical, culture médicale, maladies, épidémies et carences, nourriture et vêtements, mœurs, hygiène publique et privée, rôle des institutions laïques et ecclésiastiques, histoire des hôpitaux et des léproseries... Même si l'on ne considère que les chapitres traitant du Moyen Age, il s'agit d'un tableau large et détaillé; aucune autre région de la Suisse actuelle ne dispose, pour la période qui nous intéresse, d'une monographie aussi complète. Toutefois, Eugène Olivier mena ses recherches sans véritable contact avec les fonds d'archives, sa santé le lui interdisait; il était donc entièrement tributaire des sources imprimées ainsi que de la littérature, et dut se contenter, pour l'exploitation de sources inédites, des notes de ses nombreux correspondants. 
Incontestablement, sa performance reste digne du plus grand respect, surtout si l'on considère la situation historiographique qui précédait. Il n'y a guère que le livre d'Alice Briod, L'assistance des pauvres dans le Pays de Vaud du commencement du Moyen Age à la fin du XVI ${ }^{e}$ siècle ${ }^{2}$, qui mérite une mention. Cette thèse de droit se limitait naturellement à l'étude d'une question précise, sous l'angle juridique; ainsi, Alice Briod n'avait pas à enrichir son analyse de l'apport de recherches d'histoire familiale et sociale. Elle se basait cependant sur une excellente utilisation des sources, même inédites, avant tout les comptes d'hôpitaux. L'ouvrage garde de nos jours toute sa valeur, bien qu'il ait été écrit avant la mise au point des méthodes modernes d'investigation en matière de spiritualité et de religiosité médiévales.

L'examen de ces deux œuvres nous montre d'une certaine manière ce qui reste à faire: entreprendre des recherches systématiques dans les fonds d'archives vaudois et organiser l'étude des problèmes de santé dans un large contexte d'histoire sociale.

En ce qui concerne la documentation archivistique, nous disposons depuis quelques années du répertoire de Robert-Henri Bautier et Janine Sornay, Les sources de l'histoire économique et sociale du Moyen Age. La première partie, publiée entre 1968 et 1974, couvre notre région; elle nous permet d'entrevoir que les sources hospitalières vaudoises ${ }^{3}$ sont beaucoup plus riches qu'on pouvait le penser et tiennent la comparaison au niveau non seulement suisse, mais même européen. Pour de nombreux hôpitaux du canton de Vaud, les archives cantonales et communales nous ont conservé diverses séries de sources: comptes (Grandson, Lausanne ou Vevey), chartiers (Coppet, Lausanne, Vevey, etc.), rentiers et censiers (Moudon, Lucens, Villeneuve). Il en va de même pour d'autres institutions médiévales qui concernent de très près l'histoire de la santé: les léproseries (notamment le chartier de celle de Colovray à Nyon) et les confréries (comptes pour Nyon, rentiers et chartes en grand nombre pour Lausanne, Vevey ou Cully).

Il est inutile d'insister sur le fait qu'une documentation archivistique aussi considérable permettrait des recherches de tous ordres: histoire patrimoniale et gestion des hôpitaux, liens institutionnels et politiques, impacts sur le plan de la santé et de l'hygiène...

Aucun hôpital vaudois du Moyen Age ne pourrait sans doute être étudié sous tous ces aspects. La variété de la documentation permet cependant d'approfondir de cas en cas des domaines de recherches précis, qui pourront aisément prendre place, le moment venu, dans une vision d'ensemble. 
L'intérêt de recherches ponctuelles nous est apparu dans un certain nombre de travaux entrepris et publiés récemment, dont nous aimerions ici rendre compte rapidement.

Le même fonds d'archives d'un hôpital de Vevey, le Vieux-Mazel, a permis de traiter des aspects très différents de l'histoire d'une institution hospitalière: gestion et rentabilitié de ses vignobles au $\mathrm{XV}^{\mathrm{e}}$ siècle d'une part, approche numérique des personnes hospitalisées (1401-1416) et fonction sociale d'un hôpital médiéval d'autre part.

\section{Les comptes de l'Hôpital du Vieux-Mazel à Vevey}

\section{La rentabilité de l'exploitation du vignoble}

L'hôpital médiéval vivait pour l'essentiel du revenu de son patrimoine (droits et terres) et un peu des aumônes. Les malades n'apportaient guère de ressources: certains devenaient pensionnaires à vie en échange d'une partie de leurs biens, d'autres, recueillis par charité, avaient quelques affaires que l'hôpital vendait à leur mort. Aussi la gestion du patrimoine était-elle essentielle pour assurer le fonctionnement quotidien de l'établissement. Les recteurs du Vieux-Mazel avaient à percevoir les droits et rentes en argent ou en nature dont l'hôpital avait été gratifié; ils avaient aussi à tirer le meilleur profit des maisons et des terres qui lui appartenaient. Chaque recteur choisissait entre l'exploitation directe et la location de ces divers biens, selon les conditions économiques du moment (salaires à la hausse ou à la baisse, par exemple) et l'idée qu'il se faisait d'une bonne gestion.

Douze hectares de vignes formaient la part la plus riche de ce patrimoine; les comptes, relativement bien conservés, fournissent des données complètes pour 39 années entre le milieu du XIV ${ }^{\mathrm{e}}$ siècle et 1500 , et permettent d'en détailler la gestion. L'hôpital a toujours exploité directement une partie de ses vignes et cédé les autres contre une part de la récolte (tiers- ou mi-fruit); il était donc un gros producteur de vin. On peut suivre dans les comptes aussi bien les travaux de la vigne que les frais de vinification, ainsi que la consommation et la vente du vin. L'hôpital s'assurait en effet d'importants revenus en vendant une partie de son vin (les deux tiers); le reste servait à la consommation interne ou au versement de redevances en vin.

Les comptes sont assez précis pour permettre la reconstitution d'un bilan viticole $^{4}$, par la comparaison des frais engagés au revenu, soit fictif (produit de la vente plus valeur du vin consommé), soit réel (l'argent tiré de la vente 
du vin). Il apparaît alors que l'exploitation viticole est pour l'hôpital une source importante de bénéfices: si l'on voit dans les dépenses un investissement, le revenu représente plus de $300 \%$ des frais engagés. Si l'on déduit la consommation de l'établissement, pour ne tenir compte que du vin vendu, le revenu représente encore $180 \%$ des frais.

Une telle étude montre l'ampleur des tâches de gestion pour un hôpital médiéval, tant ses revenus dépendent des choix et du travail de son recteur, sans oublier les aléas conjoncturels (climat, prix...). Elle éclaire aussi l'importance du vin, qui n'est pas seulement une source de revenus, mais, à juger des quantités consommées, la boisson ordinaire de l'établissement; c'est d'ailleurs conforme à ce que nous savons des habitudes alimentaires du temps, où l'on mettait notamment en doute la pureté de l'eau.

\section{Hébergement et fonction d'un hôpital médiéval}

Les actes de fondation et les règlements d'hôpitaux médiévaux donnent le plus souvent la typologie des personnes qui devraient être secourues et hébergées: pauvres, pèlerins, infirmes, orphelins et malades. Il s'agit de sources qui renvoient généralement à l'image d'un hôpital idéal. La documentation hospitalière à notre disposition permet-elle de reconstituer l'hébergement en nous révélant le nombre de personnes reçues, les catégories de «malades» et la durée des séjours? Assez paradoxalement, les nombreux comptes d'hôpitaux ne nous renseignent pas directement. Ils ne livrent pratiquement jamais de renseignements quantitatifs touchant les personnes hébergées. Ce n'est que dans les cas de crise (peste, guerre ou alors jubilés entraînant un afflux extraordinaire de pèlerins), pour justifier des surcroîts de dépense ou de revenus, que des annotations comptables surgissent, portant sur les frais de décès (messes, inhumations...) ${ }^{5}$ ou, au contraire, sur la vente des objets laissés par les morts.

Toutefois, le hasard d'une recherche dans les comptes de l'Hôpital du Vieux-Mazel à Vevey nous a livré une liste exceptionnelle d'une centaine de personnes (95 pauperes) qui ont été hébergées à l'hôpital de Vevey entre 1401 et $1416^{6}$. La rareté d'une telle source, à un niveau européen, doit d'emblée être soulignée. Non seulement nous tenons là une liste presque unique de malades, mais, de plus, elle a été tenue à jour pendant cette quinzaine d'années, de sorte que nous pouvons connaître la durée effective des séjours et la date du départ des malades ou de leur mort. 
Nous disposons donc d'un assez vaste échantillon de personnes hospitalisées; toutes les catégories traditionnelles de bénéficiaires s'y rencontrent, sans qu'on puisse toutefois dire si certaines d'entre elles étaient favorisées. En effet, nous ne conservons aucun registre de délibérations qui nous renseignerait sur les refus et leurs motifs. D'autres remarques sont en revanche possibles; ainsi, bien qu'institutions vivant de leur patrimoine et donc obligées de s'en tenir à leur revenu, les hôpitaux s'adaptent mieux à l'actualité qu'on pourrait le penser : les années de grand afflux de pèlerins (les Années saintes, telles 1400, 1450,1500), celles d'épidémies ou de grande catastrophe militaire (en 1475-1476, l'armée bourguignonne campe près de Lausanne et y amène la peste; puis la ville est prise par les Suisses) voient le nombre des malades croître en flèche. C'est là le signe qu'un hôpital médiéval pouvait, au moins le temps d'une crise, rendre des services sans mesure avec sa taille et ses ressources ordinaires.

Une autre idée doit être révisée: dans le cas des hôpitaux étudiés (Vevey et Lausanne), l'essentiel des personnes hospitalisées n'est pas constitué de bourgeois de la ville qui viendraient y terminer leurs jours en échange d'une donation. Parce qu'elles enregistrent les charges obligées de l'hôpital, les sources ordinaires (chartes, préambules des comptes) mettent certes en évidence ce type particulier d'hébergement, mais, en fait, la liste de Vevey montre que les pensionnaires ne formaient pas la majorité des patients. En effet, l'hôpital abritait avant tout des personnes sans ressources et de provenances variées: un quart au moins de l'effectif n'habite ni Vevey, ni les villages alentour. L'hôpital avait même une fonction de mouroir, semble-t-il, tant les courts séjours suivis de la mort sont nombreux.

La mortalité importante (plus de $70 \%$ ) et rapide (la moitié des personnes hébergées meurent dans l'année) fait perdre de son intérêt à la discussion habituelle sur la fonction de l'hôpital et le genre de personnes accueillies : que ce soient des pensionnaires aisés, des pauvres, des malades ou des pèlerins, ce sont pour la plupart des personnes dont la survie est très souvent compromise à moyen ou à court terme.

Comme on peut s'y attendre, les secours qu'offrait l'Hôpital de Vevey étaient pour l'essentiel hôteliers et spirituels. Toutefois, une certaine activité médicale apparaît, non seulement dans l'achat de remèdes, mais dans le recours à un médecin de la ville pour soigner certains cas difficiles, telles les victimes de fractures. Enfin, lorsque l'hôpital accueillit «maître Jean, médecin, d'Avignon, juif baptisé» ${ }^{7}$, il ne faut certes pas y voir le médecin de l'établissement; c'est comme pensionnaire qu'il est reçu. Rien n'empêche 
toutefois de penser que l'on était pas indifférent à sa profession et aux services qu'il pouvait rendre.

\section{Pour une histoire des épidémies médiévales}

On entrevoit facilement les conséquences du retour de la peste en Occident, entre 1347 et 1350: énorme choc démographique immédiat, mais aussi pertes tout au long des trois siècles d'endémie qui vont suivre; bouleversements économiques et sociaux, impact sur les mentalités, réflexion intellectuelle sur la causalité, prise de mesures d'hygiène ou d'isolement...

Quelle que soit l'importance de la bibliographie générale touchant la Grande-Peste, une étude sur les épidémies de peste du $\mathrm{XIV}^{\mathrm{e}}$ siècle dans le Pays de Vaud s'imposait ${ }^{8}$. D'une part, dans des recherches conjoncturelles, l'historiographie locale ne tient pas suffisamment compte des effets de la peste, même pour une épidémie étendue à toute l'Europe, tant qu'ils n'ont pas été mis en évidence pour la région concernée. D'autre part, des données locales sûres sont moins fréquentes qu'on ne croit et en viennent rapidement à enrichir ou à modifier le tableau général qu'on a fait de la Grande-Peste. C'est ainsi que l'étude du cheminement de la peste à travers la Suisse romande a montré que c'est par là que le fléau est passé du Sud de l'Europe au Nord: les Alpes ont fait barrage et la peste a atteint l'Allemagne par Genève et le Plateau suisse.

Les sources romandes ne permettent pas de savoir si des mesures préventives ont été prises par les autorités, sauf, hélas, l'emprisonnement des Juifs, bientôt laissés sans défense devant les assauts des populations. En revanche, les statistiques de fréquence des testaments ont montré que les privés n’anticipaient pas: la plupart des testaments sont le fait de personnes déjà malades; les rares testateurs en bonne santé agissent au moment du passage de l'épidémie. Ceci, alors que la vitesse de propagation était extrêmement lente-une moyenne de $0,75 \mathrm{~km}$ par jour entre Genève et Sion-, ce qui aurait laissé le temps de réagir.

Enfin, l'étude de la mortalité est souvent aléatoire, de par la rareté des sources qui en rendent compte et l'impossibilité de connaître la population avant le passage du fléau. L'étude de petits groupes connus - moines d'un monastère, membres d'un conseil de ville... - permet de contourner cette difficulté, même si la faiblesse des effectifs considérés rend la précision des résultats fragile. En rapprochant les sources des archives vaudoises et celles 
des Archives vaticanes (qui possèdent des documents concernant la nomination de clercs dans tous les diocèses de la Chrétienté), il a été possible d'évaluer les chocs subis par le Chapitre cathédral de Lausanne en 13481349 et lors du premier retour de l'épidémie en 1360 : à chaque fois, près d'un tiers des chanoines disparaît, ce qui signifie le renouvellement presque complet de l'institution en guère plus de 10 ans. Comme rien ne prédisposait les chanoines, qui vivaient bien et séparés les uns des autres, à subir plus cruellement la peste que le reste de la population, il y a tout lieu d'extrapoler ces résultats.

\section{Histoire de la santé et institutions}

\section{Léproseries}

Une étude complète des léproseries vaudoises montre parfaitement l'importance de l'ouvrage d'Eugène Olivier et la nécessité d'entreprendre des recherches systématiques dans les différents fonds d'archives. L'ensemble des informations qui circulaient dans la littérature, souvent fort ancienne, reprises parfois par Olivier sans être vérifiées, exige un réexamen critique approfondi. En premier lieu, il est indispensable de distinguer clairement les léproseries qui ont effectivement fonctionné de celles dont l'existence est déduite de la survie d'un lieu-dit apparemment significatif («La Maladière» surtout). L'établissement d'un inventaire critique de toutes les léproseries vaudoises connues soit par la littérature soit par les archives a fait l'objet d'un mémoire de licence d'histoire de l'Université de Lausanne ${ }^{9}$; sur le territoire actuel du Canton de Vaud, il recense 28 léproseries attestées dans les sources et 38 lieux-dits.

Comme pour les hôpitaux, aucune léproserie ne peut être étudiée dans son ensemble. La découverte de plusieurs petits groupes de documents permet cependant de reconstituer des éléments essentiels de la vie d'une léproserie médiévale à partir de cas précis. Il est par exemple possible de suivre la vie d'un lépreux depuis le dépistage de sa maladie par la rumeur publique, son constat par un médecin ou les autorités locales jusqu'à l'acceptation du malade dans une léproserie. La plupart des aspects de l'organisation interne d'une telle institution ont pu être examinés, ainsi que les accords liant plusieurs communes d'une même région pour la fondation et l'entretien d'une telle institution. Quant aux attitudes de la société face aux lépreux - 
oscillant de la solidarité à l'exclusion - la documentation vaudoise est riche d'indices, notamment pour la plus grande crise dont les lépreux ont été victimes au Moyen Age: le massacre de 1321. Une importante lettre de l'évêque de Lausanne Pierre d'Oron a récemment été découverte et publiée ${ }^{10}$; à ce texte qui établit clairement que le massacre des lépreux s'est étendu du royaume de France jusqu'à Lausanne - ce que les historiens ignoraient - s'ajoutent maintenant d'autres informations : ainsi, cette même année 1321, le seigneur de la Sarraz et le prieur de Romainmôtier se disputent-ils la possession d'une léproserie, signe presque certain qu'on procédait alors à des confiscations de biens appartenant à des lépreux.

\section{Charité publique et institutions communales}

Une histoire de la santé et des institutions hospitalières doit s'appuyer sur le plus grand nombre de sources possibles. Pour Lausanne et les autres villes vaudoises, il conviendra de consulter systématiquement les registres de notaires, les manuaux des conseils et les comptes communaux.

Elargir la base documentaire est nécessaire pour prendre en considération des aspects jusqu'ici négligés. Un ouvrage récent sur les attitudes des Lausannois du $\mathrm{XIV}^{\mathrm{e}}$ siècle face à la mort ${ }^{11}$ a bien montré l'existence de liens profonds de solidarité entre les laïcs lausannois et les hôpitaux de leur ville. L'analyse des legs pieux montre que, tout au long du siècle, plus de $60 \%$ des testateurs favorisent un hôpital. Au même titre que la paroisse et les confréries, l'hôpital fait partie de l'imaginaire du testateur. On voit à quel point, au XIV e siècle déjà, les hôpitaux, dont l'histoire était somme toute relativement récente, sont désormais un élément fondamental de la vie urbaine. Toutefois, la Grande-Peste marque une coupure : on passe de $82 \%$ de legs à l'hôpital jusqu'en 1350 à $45 \%$ pour la seconde moitié du siècle. Il ne faut d'ailleurs pas y voir une désaffection pour cette institution: la Peste a entraîné une concentration des legs sur la paroisse du testateur-paroisse qui va recueillir son corps et entretenir son souvenir - au détriment des autres bénéficiaires traditionnels des dispositions charitables.

Dans une société en train de gagner de nouveaux espaces de gestion communale de la chose publique, l'hôpital se trouva de plus en plus au centre des rapports entre le seigneur de la ville (l'évêque) et la commune de Lausanne. Dès la fin du $\mathrm{XIII}^{\mathrm{e}}$ siècle, le droit à la gestion de l'hôpital faisait partie des aspirations des Lausannois à une organisation communale 
reconnue. Le premier document répartissant, en 1282, la gestion de l'Hôpital Notre-Dame de Lausanne entre évêque, chanoines et bourgeois, appartient justement aux lendemains de la plus ancienne tentative d'instaurer une commune dans la ville. Ce n'est cependant qu'en 1528 que les Lausannois réussiront à arracher à l'Eglise le contrôle complet de cet hôpital ${ }^{12}$. Dans les mêmes années, l'abbaye des nobles enfants de Lausanne, étroitement liée au pouvoir communal, s'empare de l'autre hôpital de Lausanne, celui de SaintJean, dépendant des chanoines du Grand-Saint-Bernard. En 1533, l'évêque se plaint de ne pas être parvenu à les en déloger ${ }^{13}$.

Pour Lausanne comme pour le reste du pays, seule une étude détaillée de la documentation, pratiquement inexplorée, permettra de suivre l'importance que le contrôle administratif et économique d'un hôpital revêtit progressivement dans l'affirmation du pouvoir municipal. En s'occupant de l'hôpital, le personnel politique des villes du bas Moyen Age a fait l'apprentissage de la gestion publique. 


\section{Notes}

1 La partie concernant les XVII ${ }^{e}$ et $\mathrm{XVIII}^{e}$ siècles est parue en 1939 ; la première partie - des origines au XVII siècle - a été publiée en 1962 par une équipe d'historiens à partir des papiers d'Olivier, tandis que le solde de l'édition de 1939 était remis en vente sous une autre jaquette. L'ensemble forma dès lors les tomes 29 à 32 de la Bibliothèque historique vaudoise.

2 Lausanne 1926; réédition, mais sans les notes, Lausanne 1976.

3 I/2, Paris 1971, p. 902-903, 919-920 et 937-943.

4 Monselesan Cynthia, Les vignes de l'Hôpital de Vevey au XV' siècle: rentabilité d'une exploitation, à paraître dans Revue historique vaudoise, 98 (1991).

5 Morerod Jean-Daniel, Jubilés et passages de pèlerins : les hôpitaux de Vevey et de Lausanne au XV' siècle, dans: Ceux qui passent et ceux qui restent. Etudes sur les trafics transalpins et leur impact local (Actes du colloque de Bourg-Saint-Pierre, 1988). Editions du bi-millénaire du Grand-Saint-Bernard 1989, p. 115-130.

6 Morerod Jean-Daniel et Paravicini Bagliani Agostino, Une liste des malades de l'hôpital de Vevey (1401-1416), à paraître dans Revue historique vaudoise, 98 (1991).

7 On est tenté de l'identifier avec Mö̈se Ben Samuel, médecin juif de Roquemaure, converti sous le nom de Jean d'Avignon, et célèbre pour son activité en Espagne dans la seconde moitié du XIV" siècle; v. Wickersheimer Ernest et JAQuart Danielle, Dictionnaire biographique des médecins en France au Moyen Age, Genève 1979, t. 1, p. 356, et t. 3, p. 143.

8 Andenmatten Bernard et Morerod Jean-Daniel, La Peste à Lausanne au XIV siècle $(1348 / 49,1360)$. Etude du Chapitre cathédral et des testaments vaudois, dans Etudes de Lettres, 2-3, 1987, p. 19-49.

9 Borradori Piera, La Lèpre dans le Pays de Vaud: Histoires d'exclusion et de solidarité, mars 1991 (dactyl.). Il paraîtra prochainement dans les Cahiers lausannois d'Histoire médiévale.

10 Morard Nicolas, A propos d'une charte inédite de l'évêque Pierre d'Oron: lépreux brûlés à Lausanne en 1321, dans Revue d'Histoire ecclésiastique suisse, 75 (1981), p.231-238.

11 Pasche Véronique, «Pour le salut de mon âme». Les Lausannois face à la mort (XIV" siècle), Lausanne 1989 (Cahiers lausannois d'Histoire médiévale, 2).

12 Grandjean Marcel, Les Monuments d'art et d'Histoire de la Suisse, Canton de Vaud, t. I, Bâle 1965, p. 298-299, et t. IV, Bâle 1981, p.399-401. V. aussi t. I, p.313-320, pour les autres hôpitaux et léproseries de Lausanne au Moyen Age.

13 Taddei Ilaria, Fêtes, Jeunesse et Pouvoirs. L'Abbaye des nobles enfants de Lausanne, Mémoire de la Faculté des Lettres de Lausanne, mars 1991 (dactyl.), p. 26-27 et 95. Il paraîtra prochainement dans les Cahiers lausannois d'Histoire médiévale. 


\section{Summary}

\section{History of health in the Country of Vaud in the Middle Ages}

The rich archival sources of mediaeval Vaudois institutions make it possible to study a great variety of health problems of that country. Recent studies elucidate, e.g., the administration of the vineyards of the hospital of the town of Vevey as well as the management of great numbers of pilgrims or victims of a disaster by the same hospital. Progression and demographic consequences of the plague outbreaks of 1348 and 1360 have been worked out and the network of leper-houses could be reconstructed. Finally, the ambiguous attitude of the citizens of Lausanne towards their hospital in the 14 th century has been revealed: pious legacies on the one hand, endeavour to control them politically on the other.

\section{Zusammenfassung}

\section{Die Geschichte des Gesundheitswesens des Waadtlands im Mittelalter}

Die reichen Akten der mittelalterlichen Institutionen der Waadt lassen die verschiedensten Aspekte des Gesundheitswesens untersuchen. Neuere Studien behandeln die Verwaltung der Rebberge des Spitals von Vevey, sie gehen den zu Beginn des 15. Jahrhunderts hier hospitalisierten Personen nach und zeigen, wie das Hospital mit dem Massenandrang von Pilgern und Katastrophenopfern fertig wurde. Ausbreitung und demographische Auswirkungen der Pestzüge von 1348 und 1360 konnten präzisiert, das Netz der waadtländischen Leprosorien rekonstruiert werden. Schliesslich offenbart sich die zwiespältige Einstellung der Bürger von Lausanne gegenüber ihrem Spital in frommen Vergabungen einerseits, im Streben nach politischer Kontrolle andererseits.

J.-D. Morerod et A.Paravicini Bagliani

Faculté des Lettres

Université de Lausanne

BFSH 2

CH-1015 Lausanne 